\title{
Relationship between blood pressure levels and bone mineral density in postmenopausal Turkish women
}

Selma Yazici', Mehmet Yazici², Ugur Korkmaz³, Melih Engin Erkan, Ali Erdem Baki², Ismail Erden², Hakan Ozhan², Safinaz Ataoğlu

1Department of Physical Medicine and Rehabilitation, Düzce University Medical Faculty,
Düzce, Turkey
2Department of Cardiology, Düzce University Medical Faculty, Düzce, Turkey
3Department of Gastroenterology, Kocaeli University Medical Faculty, Kocaeli, Turkey
4Department of Nuclear Medicine, Düzce University Medical Faculty, Düzce, Turkey

Submitted: 27 March 2010

Accepted: 9 September 2010

Arch Med Sci 2011; 7, 2: 264-270

DOI: 10.5114/aoms.2011.22077

Copyright (c) 2011 Termedia \& Banach

\section{Abstract}

Introduction: We investigated the association between bone mineral density (BMD) detected by dual-energy X-ray absorptiometric (DXA) method and blood pressure (BP) in a large sample of postmenopausal women.

Material and methods: The current study was based on a retrospective analysis of 586 postmenopausal women with a mean age of $60.8 \pm 8.8$ years, who were screened for osteopenia or osteoporosis by DXA. Patients with hypertension ( $\mathrm{HT}, n=306$ ) were compared with normotensive (NT, $n=290$ ) individuals. Bone mineral density results for the femur neck and spine were classified into 3 groups according to World Health Organization criteria: normal (T score $>-1.0$ SD), osteopenia (T score -1.0 to -2.5 SD) and osteoporosis (T score $<-2.5$ SD). Patients with osteopenia or osteoporosis (T score $<-1.0$ SD) were grouped as having low bone mass (LBM).

Results: There were no significant differences in femur T score, femur BMD, femur Z score, spinal T score, spinal BMD and spinal Z score between hypertensive and normotensive groups. The group of patients with low bone mass calculated from femur T scores had higher age, systolic BP, duration of hypertension and duration of menopause, but lower BMI. Similarly, patients with low spine BMD had higher age and duration of menopause, but lower BMI. Linear regression analysis showed a significant correlation between systolic BP and femur BMD and T score values. Furthermore, logistic regression analysis revealed that hypertension is an independent predictor of spinal osteopenia and osteoporosis.

Conclusions: The presence of hypertension is an independent predictor of spinal low bone density in Turkish women after menopause.

Key words: blood pressure, bone mineral density, dual-energy X-ray absorptiometric.

\section{Introduction}

The association between high blood pressure (BP) and abnormalities in calcium metabolism has been previously investigated in experimental, clinical and epidemiological studies but the existing evidence is inconclusive [1-6]. In several studies it has been consistently reported that sustained hypercalciuria as a result of high BP may be the underlying mechanism that explains the pathophysiology of hypertensive bone loss [7-9].

\author{
Corresponding author: \\ Selma Yazici, MD \\ Düzce University \\ Medical Faculty \\ Department of Physical \\ Medicine and Rehabilitation \\ Düzce, Turkey, PC: 81620 \\ Phone: 0090-380 541407 \\ Fax: 00903805414213 \\ E-mail: \\ drselmayazici@yahoo.com.tr
}


Furthermore, hypertension is presumed to be related to alterations in calcium metabolism leading to increased calcium losses, secondary activation of the parathyroid gland, and increased movement of calcium from bone [1, 4]. Such long-lasting disturbances in calcium metabolism may constitute one of the mechanisms involved in the development of excessive age-related BMD reduction. In addition, it has been suggested that calcium loss related to high BP may be due to a defect in the kidney's ability to handle calcium. Finally, some studies have also suggested that increased angiotensin II levels in hypertensive patients have a harmful effect by increasing bone resorption and inhibiting mineralization [10-12]. Consistent with these results, in some studies, intake of calcium has been shown to correlate inversely with BP and to have a beneficial effect in human hypertension $[13,14]$. In a large cohort of British women, Cappuccio et al. found that femoral neck BMD detected by the dual-energy X-ray absorptiometric (DXA) method was inversely related to the highest tertile of systolic and diastolic BP values [4]. However, Mussolino et al. reported that there were no significant associations between BMD and hypertension in men or women of African American or white ethnicity by using the National Health and Nutrition Examination Survey (NHANES) data [15].

To the best of our knowledge, the association between blood pressure and bone mass has not been studied previously in the Turkish population. Therefore, in a large national sample of postmenopausal women in whom both osteoporosis and hypertension are highly prevalent, we aimed to determine femoral neck and $L_{2}-L_{4}$ spine BMD values detected by the DXA method and compare these parameters in normotensive and hypertensive groups.

\section{Material and methods}

\section{Population sample}

The current study, which was carried out in the departments of Cardiology and Physical Medicine of Düzce University Faculty of Medicine, was based on a retrospective analysis of 750 consecutive individuals screened for osteopenia or osteoporosis by dual energy X-ray absorptiometry (DXA) between February 2005 and May 2008. In the final analytic sample, 586 postmenopausal women (with a mean age of $60.8 \pm 8.8$ years) without any exclusion criteria were included in the study. Exclusion criteria were known chronic infectious or inflammatory disease, known coronary heart disease, thyroid diseases, severe liver or renal disease, neoplasm, haematological disorders, taking anticoagulant therapy, and physical inactivity. In addition, patients taking hormone replacement therapy (HRT) were also excluded. Postmenopausal status was determined by a "no" response to the question, "Have you had a period in the past 12 months?" Prior medical histories, including comorbidities that might affect bone density, were recorded. Approximately one out of five patients in each group have been treated with alendronate $(70 \mathrm{mg})$, risedronate $(35 \mathrm{mg})$ or raloxifene $(60 \mathrm{mg})$ along with calcium (1000 mg) and vitamin D (0.5 $\mu \mathrm{g})$. Diuretic, antihypertensive agents and steroid use, known to affect bone mass, were also recorded. Anthropometric and biochemical data were collected from the patients' physical medicine files and laboratory records.

The Institutional Review Board of the Düzce University Faculty of Medicine approved the study, a waiver of consent was granted and patient identity was protected.

\section{Measurements of risk variables}

Hypertension was defined as blood pressure $\geq 140 \mathrm{mmHg}$ and/or $\geq 90 \mathrm{mmHg}$, and/or use of antihypertensive medication [16]. Blood pressure was measured in the sitting position on the right arm, and the mean of two recordings at least 3 min apart was recorded. Body mass index (BMI) was computed as weight divided by height squared $\left(\mathrm{kg} / \mathrm{m}^{2}\right)$. In a combined assessment of leisure-time and work activity, during last year, it was categorized into four grades of increasing activity via a questionnaire in which the participant, assisted by the survey physician, assigned a grade for her activity. The four levels were: (1) being almost entirely inactive or engaging in light physical activity, 2 h per week (e.g. reading, watching television); (2) engaging in light physical activity for 2-4 h per week (e.g. walking, cycling, washing dishes, light gardening, light physical exercise); (3) engaging in light physical activity for $4 \mathrm{~h}$ per week or more vigorous activity for 2-4 h per week (e.g. brisk walking 3-4 mph, fast cycling, heavy gardening, sports that cause perspiration or exhaustion); and (4) engaging in vigorous physical activity for $4 \mathrm{~h}$ per week, regular heavy exercise, or competitive sports several times per week. Level 1 was considered physically inactive, level 2 was considered a low level of physical activity, and levels 3 and 4 together were considered a moderate to high level of physical activity [17].

\section{Bone mineral density scans}

Bone mineral density $\left(\mathrm{g} / \mathrm{cm}^{2}\right)$ was measured for the lumbar spine $\left(L_{2}\right.$ to $\left.L_{4}\right)$ and proximal femur using a GE Lunar DPX-NT PRO (Lunar Corp, Adison, WI, USA) by DXA scanning. Bbone mineral density was determined according to standard Lunar 
protocols. Bone mineral density was expressed in $\mathrm{g} / \mathrm{cm}^{2}$ and as peak bone mass percentage in normal subjects (T-score) depending on the software used in the device. Results for the femur neck and lumbar spinal were classified into 3 groups according to World Health Organization criteria: normal (T score >-1.0 SD), osteopenia (T score -1.0 to -2.5 SD) and osteoporosis (T score $<-2.5 \mathrm{SD}$ ). Patients with osteopenia or osteoporosis ( $T$ score $<-1.0$ SD) were grouped as having low bone mass.

\section{Statistical analysis}

All continuous variables were tested for skewness. The distribution of all variables was normal. The continuous parameters were presented as mean \pm standard deviation. Comparisons of demographic, clinical and laboratory characteristics of the patients were performed using independent sample $t$-test or one-way ANOVA test for continuous variables. Categorical variables were compared with the $\chi^{2}$ test. Logistic regression model with enter method was used to evaluate associations between hypertension and femur BMD or spine BMD. Linear regression analysis was performed to examine the association between $T$ scores and bone mass covariates including hypertension. Multivariate logistic regression analysis was used to assess the independent association of BMD with hypertension. Statistical analyses were carried out using SPSS 13.0 for Windows and a $p$-value $<0.05$ was considered significant

\section{Results}

In total, 586 subjects (mean age $60.8 \pm 8.8$ years, BMI $30.9 \pm 5.3 \mathrm{~kg} / \mathrm{m}^{2}$ ) were analysed. $52.2 \%$ of them had hypertension. Demographic, clinical and some laboratory variables of the subjects with and without HT are shown in Table I. The number of patients taking different agents of treatment for osteoporosis was similar in both groups (Table I). Hypertensive patients were older and had significantly higher BMI, duration of menopause, and systolic and diastolic BP values. Bone mineral density variables were also similar between the two groups (Table I).

Linear regression analysis showed that hypertension, age, BMI, smoking status and physical activity were significant correlates of femur or spinal BMD and T scores (Figure 1). Furthermore, logistic regression analysis was used to identify independent determinants of LBM (Table II). The covariates considered were hypertension, age, physical activity, and smoking status and BMI. Hypertension was found to be an independent predictor of LBM (osteoporosis and osteopenia) measured from the spine. On the other hand, only age and BMI were left as independent predictors of LBM measured from the femur neck.

Moreover, the study population was further divided into two groups as normal $(n=224)$ and low bone mass (LBM) according to femur bone mass and/or spine bone mass values. When compared with normal bone mass (NBM) for femur, the group with LBM $(n=362)$ had higher age, systolic BP, duration of hypertension, duration of menopause and number of births, but lower BMI (Figure 2). Similarly, when compared with NBM for spine $(n=182)$, the group with LBM $(n=403)$ had higher age, duration of menopause and number of births, but lower BMI. However, no independent relationship was found between these parameters and systolic or diastolic BP in logistic regression analysis.

\section{Discussion}

In the present study, femur BMD, femur T score, femur $Z$ score, spine BMD, spine T score and spine $Z$ score values were similar in both normotensive and hypertensive groups. Hypertension was found to be an independent predictor of osteoporosis and osteopenia measured from the spine. When BMD was measured from the femur neck, only age and BMI were left as independent predictors of LBM.

Trabecular bone (lumbar spine, os calcis) has been shown to be more sensitive to metabolic changes compared with cortical bone (femoral neck, distal radius) [18]. The association between hypertension and osteopenia has been shown to be site specific [19-21]. Furthermore, there are differences in the timing of bone loss in healthy women, with trabecular bone diminishing with every decade of life but cortical bone levels being similar in the third, fourth, and fifth decades [22]. As there are essential differences in response to environmental (including hormonal or nutritional) factors between the cortical and trabecular part of bone structure, as in our study, it would seem more logical that measurement of both cortical (at the femoral neck) and trabecular bone (lumbar spine) is necessary.

The link between hypertension and abnormalities in calcium metabolism that lead to increased calcium loss from bone in adults has been studied previously but the results differ according to the ethnic groups and are inconclusive [4, 5, 10, 13, 19-24]. Tsuda et al. compared the BMD of 31 hypertensive Japanese women to 14 normotensives and reported inverse correlations between lumbar spine BMD and systolic blood pressure (SBP) [23]. In another study, Gotoh et al. reported that lumbar spine BMD provides an index of whole calcium balance, and implied that elevated blood pressure is associated with abnormalities in calcium 
Table I. Comparisons of demographic, clinical and laboratory characteristics of groups

\begin{tabular}{|c|c|c|}
\hline Parameters & $\begin{array}{l}\text { Normotensive subjects } \\
\qquad(n=280)\end{array}$ & $\begin{array}{l}\text { Hypertensive patients } \\
\qquad(n=306)\end{array}$ \\
\hline Age [years] & $58.9 \pm 8.6$ & $62.4 \pm 8.7^{\ddagger}$ \\
\hline Body mass index [kg/m²] & $30.2 \pm 5.2$ & $31.5 \pm 5.3^{\dagger}$ \\
\hline Systolic blood pressure [mmHg] & $121.0 \pm 11.4$ & $149.4 \pm 15.3^{\ddagger}$ \\
\hline Diastolic blood pressure [mmHg] & $76.3 \pm 8.2$ & $90.4 \pm 9.1^{\ddagger}$ \\
\hline Serum calcium [mg/dl] & $9.5 \pm 0.5$ & $9.4 \pm 0.5$ \\
\hline Serum phosphorus [mg/dl] & $3.7 \pm 0.5$ & $3.8 \pm 0.7$ \\
\hline Bone mineral density (BMD) femur $\left[\mathrm{g} / \mathrm{cm}^{2}\right]$ & $0.83 \pm 0.12$ & $0.82 \pm 0.12$ \\
\hline Femur T-score & $-1.16 \pm 1.02$ & $-1.24 \pm 1.03$ \\
\hline Femur Z-score & $-0.21 \pm 0.94$ & $-0.08 \pm 0.82$ \\
\hline Bone mineral density (BMD) spine $\left[\mathrm{g} / \mathrm{cm}^{2}\right]$ & $0.95 \pm 0.16$ & $0.94 \pm 0.18$ \\
\hline Spine T-score & $-1.51 \pm 1.31$ & $-1.59 \pm 1.42$ \\
\hline Spine Z-score & $-0.65 \pm 1.28$ & $-0.52 \pm 1.31$ \\
\hline Low bone density for femur [\%] & 56.8 & $66.4^{4}$ \\
\hline Low bone density for spine [\%] & 66.1 & 71.2 \\
\hline Blood pressure medication [\%] & - & 38 \\
\hline Current smokers [\%] & 11.1 & 5.9 \\
\hline Package-years & $15.7 \pm 12.6$ & $12.5 \pm 7.8$ \\
\hline \multicolumn{3}{|l|}{ Physical activity level [\%] } \\
\hline Low & 44.7 & 42.8 \\
\hline Moderate & 48.6 & 50.0 \\
\hline High & 5.4 & 6.9 \\
\hline Duration of hypertension [years] & - & $7.7 \pm 6.8$ \\
\hline Years since menopause & $12.1 \pm 9.4$ & $15.3 \pm 9.1^{\Perp}$ \\
\hline Presence of diabetes mellitus [\%] & 14.3 & 16.4 \\
\hline Therapy for osteoporosis [\%] & 22.8 & 21.9 \\
\hline Alendronate use $(n)$ & 49 & 45 \\
\hline Risedronate use $(n)$ & 12 & 18 \\
\hline Raloxifene use $(n)$ & 3 & 4 \\
\hline
\end{tabular}

$\neq p<0.001$, vs. normotensive group, $\dagger^{\dagger} p<0.005$, vs. normotensive group, $₫ p<0.05$, vs. normotensive group
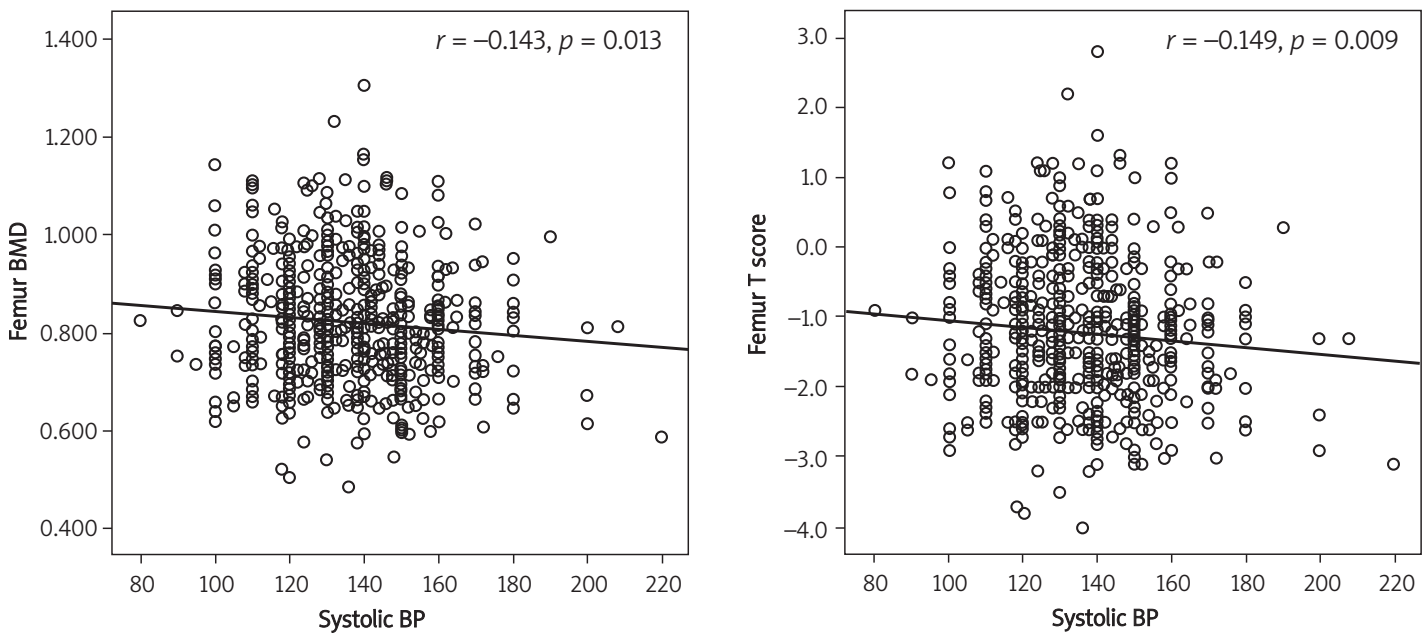

Figure 1. Associations between femur BMD and T scores of hypertensive subjects and systolic blood pressure (BP) values in linear regression analysis 
Table II. Independent predictors of osteoporosis and osteopenia (according to BMD values measured from the spine) in multivariate logistic regression analysis

\begin{tabular}{|c|c|c|c|c|}
\hline \multicolumn{5}{|c|}{ Independent predictors of osteoporosis } \\
\hline \multirow[t]{2}{*}{ Parameters } & \multirow[t]{2}{*}{ OR } & \multicolumn{2}{|c|}{$95 \%$ Confidence interval } & \multirow[t]{2}{*}{ Value of $p$} \\
\hline & & Lower & Upper & \\
\hline Age & 1.079 & 1.052 & 1.107 & $<0.001$ \\
\hline Body mass index & 0.91 & 0.876 & 0.945 & $<0.001$ \\
\hline Smoking status & 0.473 & 0.246 & 0.412 & 0.025 \\
\hline Physical activity & 1.455 & 0.99 & 2.139 & 0.057 \\
\hline Presence of hypertension & 0.532 & 0.355 & 0.798 & 0.002 \\
\hline \multicolumn{5}{|c|}{ Independent predictors of osteopenia } \\
\hline \multirow[t]{2}{*}{ Parameters } & \multirow[t]{2}{*}{ OR } & \multicolumn{2}{|c|}{$95 \%$ Confidence interval } & Value of $p$ \\
\hline & & Lower & Upper & \\
\hline Age & 1.079 & 1.055 & 1.105 & $<0.001$ \\
\hline Body mass index & 0.904 & 0.868 & 0.941 & $<0.001$ \\
\hline Smoking status & 0.752 & 0.435 & 1.639 & 0.473 \\
\hline Physical activity & 1.548 & 1.05 & 2.281 & 0.027 \\
\hline Presence of hypertension & 0.61 & 0.408 & 0.912 & 0.016 \\
\hline
\end{tabular}
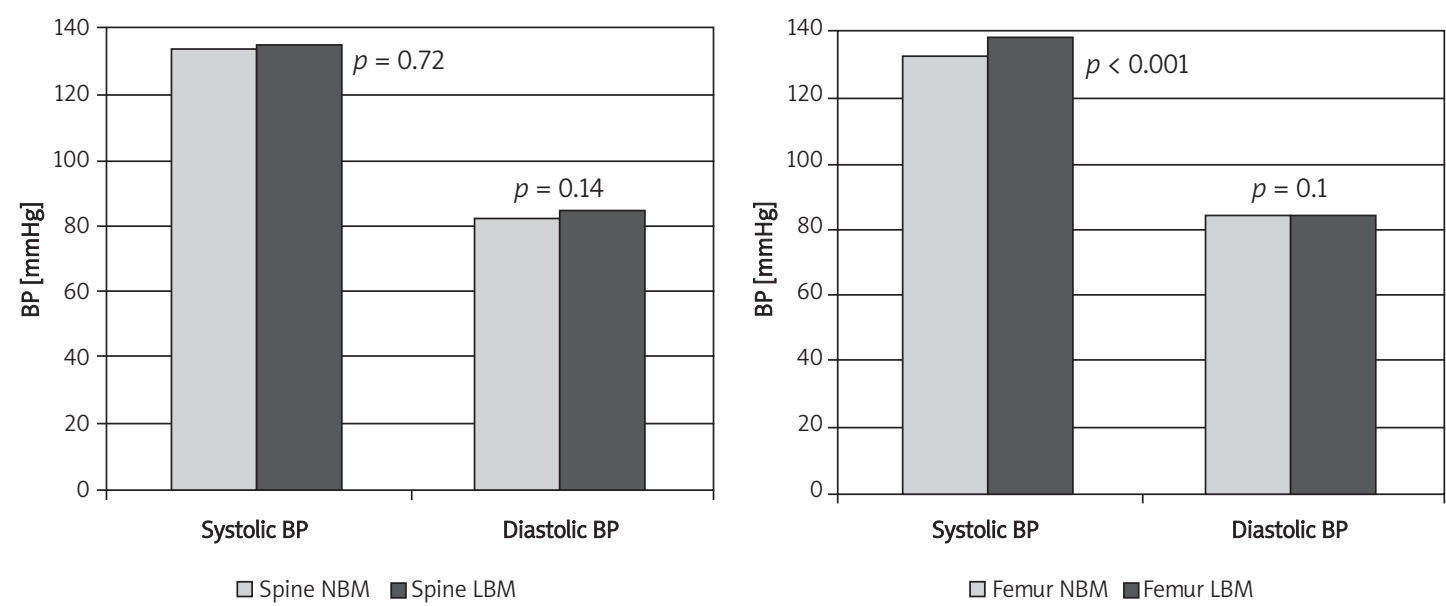

Figure 2. Comparisons of blood pressure values in groups (according to the measurements of femur or spine bone mineral density)

metabolism [13]. Similarly, Afghani et al. reported that hypertensive women had significantly lower bone mineral content (BMC) compared with normotensive women and SBP was significantly, independently and inversely related to BMC, but not BMD, in premenopausal women [22]. Their analysis was restricted to a small population of Hispanic women (9 hypertensive and 24 normal) and the findings may not be generalizable to other ethnic groups. In a large British cohort, Cappuccio et al. studied the relation between bone mass and blood pressure in white postmenopausal elderly women. They found femoral neck BMD to be inversely related to systolic and diastolic blood pressure, but only with the highest BP quartile [4].
Contrary to our study, they also restricted their analysis to the neck of the femur, so they could not conclude about this association at different sites. Likewise, a population-based study of 6886 Swedish women (aged 50 to 59 years) reported that bone density was positively associated with systolic BP. Lidfeldt et al. revealed that the relationship was most evident among postmenopausal women without hormone replacement therapy. However, they did not control for potential confounders such as BMI [5]. Our findings are concordant with these results.

Contrary to these findings, Mussolino et al. reported that no relationship existed between hypertension and proximal femur BMD after 
controlling for body mass index and other potential confounders (age, smoking, alcohol consumption, physical activity and so on) in one of a few largescale studies of hypertension and BMD [15]. Moreover, they previously reported that there was no significant association between BMD and hypertension in the NHANES study [25]. It is clear that diagnosis of LBM changes according to the site of DXA analysis. In these studies the authors only measured BMD from the femur neck and they could not find any association between hypertension and BMD. In our study, hypertension was found to be an independent predictor of LBM measured only from the spine.

Sustained hypercalciuria as a result of high BP was speculated as the underlying mechanism that explains the pathophysiology of hypertensive bone loss [7-9]. Primary hypertension is related to alterations in calcium metabolism leading to increased calcium losses, secondary activation of the parathyroid gland, and increased movement from bone [1-5]. Such long-lasting disturbances in calcium metabolism may constitute one of the mechanisms involved in the development of excessive age-related BMD reduction. If these disturbances persist for many years, they may additionally promote female senile osteopenia. On the other hand, Perez-Castrillon et al. proposed that because increased angiotensin II levels have a harmful effect on bone mass by increasing bone resorption and inhibiting mineralization, angiotensin-converting enzyme (ACE) inhibitors or angiotensin II-receptor blockers may be useful in the treatment of osteoporosis in hypertensive women [26]. By contrast, the number of hypertensive subjects who have been taking antihypertensive drugs such as thiazides and ACE inhibitors, which could positively interfere with calcium metabolism, was not specified in the analysis of many studies $[26,27]$.

These conflicting results in previously reported studies might be explained at least in part by different patient selections (such as age, ethnicity, sample size, inclusion of patients with comorbidities, different extent and aetiology of osteoporosis or hypertension) and different diagnostic methods or criteria. In the present study, we applied strict rules in the selection of the study population and excluded patients with confounding factors, such as HRT use, especially affecting bone mass.

Approximately one out of five subjects in each group had been treated for osteoporosis with various medications, all of which are believed to have different effects on the patient's BMD. It is possible that the heterogeneity of the two groups would confound the results of the study. Our analysis is restricted to postmenopausal women aged 50 years and older; therefore, the findings are not generalizable to men or younger women. In addition, a source of possible bias is misclassification because of assessment of hypertension at a single visit in the present study (as in the NHANES III study). Particularly, it should be emphasized that the cross-sectional design of this study precludes any conclusions on cause-effect relationships between blood pressure and bone mineral status in women. Therefore, a prospective designed study which has a larger sample size in addition to the measurement of biochemical indices of bone mineral metabolism would need to be employed to evaluate in more detail the relationship between $\mathrm{BP}$ and BMD.

In conclusion, the present study is the first to evaluate the association between hypertension and bone mineral density in postmenopausal Turkish women. The presence of hypertension was found to be an independent predictor of low bone density.

\section{Acknowledgments}

The authors thank our laboratory technicians for DXA scanning.

\section{References}

1. McCarron DA, Pingree PA, Rubin RJ, Gaucher SM, Molitch M, Krutsik S. Enhanced parathyroid function in essential hypertension: a homeostatic response to a urinary calcium leak. Hypertension 1980; 2: 162-8.

2. Izawa Y, Sagara K, Kadata T, Makita T. Bone disorders in spontaneously hypertensive rats. Calcif Tissue Int 1985; 37: 605-7.

3. Cirillo M, Galletti F, Strazzullo P, Torielli L, Melloni MC. On the pathogenetic mechanism of hypercalciuria in genetically hypertensive rats of the Milan strain. Am J Hypertens 1989; 2: 741-6.

4. Cappuccio FP, Meilahn E, Zmuda JM, Cauley JA. High blood pressure and bone-mineral loss in elderly women: a prospective study. Lancet 1999; 354: 971-5.

5. Lidfeldt J, Holmdahl L, Samsioe G, et al. The influence of hormonal status and features of the metabolic syndrome on bone density: a population-based study of Swedish women aged 50 to 59 years. The Women's Health in the Lund Area Study. Metabolism 2002; 51: 267-70.

6. McGrowder D, Williams A, Gordon L, et al. Hypocalciuria in pre-eclampsia and gestational hypertension due to decreased fractional excretion of calcium. Arch Med Sci 2009; 5: 80-5.

7. Strazzullo P, Nunziata V, Cirillo M, et al. Abnormalities of calcium metabolism in essential hypertension. Clin Sci 1983; 65:137-41.

8. Strazzullo P. The renal calcium leak in primary hypertension: pathophysiological aspects and clinical implications. Nutr Metab Cardiovasc Dis 1991; 1: 98-103.

9. Cappuccio FP, Kalaitzidis R, Duneclift S, Eastwood JB. Unravelling the links between calcium excretion, salt intake, hypertension, kidney stones and bone metabolism. J Nephrol 2000; 13: 169-77.

10. Perez-Castrillon JL, Justo I, Silva J, et al. Bone mass and bone modelling markers in hypertensive postmenopausal women. J Hum Hypertens 2003; 17: 107-10. 
11. Hatton R, Stimpell M, Chambers TJ. Angiotensin II is generated from angiotensin I by bone cells and stimulates osteoclastic bone resorption in vitro. J Endocrinol 1997; 152: 5-10.

12. Hiruma Y, Inoue A, Hirose S, Hagiwara H. Angiotensin II stimulates the proliferation of osteoblast-rich populations of cells from rat calvariae. Biochem Byophys Res Commun 1997; 230: 176-8.

13. Gotoh M, Mizuno K, Ono Y, Takahashi M. High blood pressure, bone-mineral loss and insulin resistance in women. Hypertens Res 2005; 28: 565-70.

14. Belizan JM, Villar J, Gonzales L, Campodonico L, Bergel E. Calcium supplementation to prevent hypertensive disorders of pregnancy. N Engl J Med 1991; 325: 1399-405.

15. Mussolino ME, Madans JH, Gillum RF. Bone mineral density and hypertension prevalance in postmenopausal women: result from Third National Health and Nutrition Examination Survey. Ann Epidemiol 2006; 16: 395-9.

16. Chobanian AV, Bakris GL, Black HR, et al.; National Heart, Lung, Blood Institute; National High Blood Pressure Education Program Coordinating Committee. Seventh report of the Joint National Committee on Prevention, Detection, Evaluation, and Treatment of High Blood Pressure. Hypertension 2003; 42: 1206-52.

17. Pedersen JO, Heitmann BL, Schnohr P, Grønbæk M. The combined influence of leisure-time physical activity and weekly alcohol intake on fatal ischaemic heart disease and all-cause mortality. Eur Heart J 2008; 29: 204-12.

18. Tsurusaki K, Ito M, Hayashi K. Differential effects of menopaus and metabolic disease on trabecular and cortical bone assessed by peripheral quantitative computed tomography (pQCT). Br J Radiol 2000; 73: 14-22.

19. Metz JA, Morris CD, Roberts LA, McClung MR, McCarron DA. Blood pressure and calcium intake are related to bone density in adult males. Br J Nutr 1999; 81: 383-8.

20. Jankowska EA, Susanne C, Roguckay E, Medras M. The inverse relationship between bone status and blood pressure among Polish men. Ann Hum Biol 2002; 29: 63-73.

21. Larijani B, Bekheirnia MR, Soltani A, Khalili-Far A, Adibi H, Jalili RB. The bone mineral density is related to blood pressure in men. Am J Hum Biol 2004; 16: 168-71.

22. Afghani A, Johnson CA. Resting blood pressure and bone mineral content are inversly related in overweight and obese Hispanic women. Am J Hypertens 2006; 19: 286-92.

23. Tsuda K, Nishio I, Masuyama Y. Bone mineral density in women with essential hypertension. Am J Hypertens 2001; 14: 704-7.

24. Buchanan JR, Myers C, Lloyd T, Greer RB III. Early vertebral trabecular bone loss in normal premenopausal women. J Bone Miner Res 1988; 3: 583-7.

25. Mussolino ME, Madans JH, Gillum RF. Bone mineral density, blood pressure, and stroke in elderly women. Stroke 2003; 34: e210-1.

26. Perez-Castrillon JL, Justo I, Silva J, et al. Relationship between bone mineral density and angiotensin converting enzyme polymorphism in hypertensive postmenopausal women. Am J Hypertens 2003; 16: 233-5.

27. Lynn H, Kwok T, Wong SY, Woo J, Leung PC. Angiotensin converting enzyme inhibitor use is associated with higher bone mineral density in elderly Chinese. Bone 2006; 38: 584-8. 